\title{
Dyspnea Management in Patients with Cancer
}

\author{
Zehra GÖK METiN, Ayşe ARIKAN DÖNMEZ
}

Department of Internal Medicine Nursing, Hacettepe University Faculty of Nursing, Ankara-Turkey

\begin{abstract}
SUMMARY
Dyspnea in patients with cancer is a common symptom and is generally associated with anxiety. It limits patients' activities of daily living and adversely affects not only patients, but also caregivers. It remains a difficult symptom to manage, despite recent advances in cancer treatment and the increasing evidence available. Different pharmacological approaches such as opioids, and nonpharmacological interventions including oxygen, fun, exercise, pulmonary rehabilitation, acupuncture, acupressure and cognitive-behavioral therapies are used to manage dyspnea. Symptom management and supportive therapies given by oncology nurses become important in patients with cancer who experience dyspnea. There are limited number of publications about the management of dyspnea in Turkish oncology nursing literature. This literature review aims to inform nurses about cancer patients with dyspnea.
\end{abstract}

Keywords: Cancer; dyspnea; management; nursing.

Copyright $\odot$ 2016, Turkish Society for Radiation Oncology

\section{Introduction}

The definition, underlying pathophysiological causes, and identification of dyspnea will be discussed in the introduction section of this review article. Pharmacological and non-pharmacological approaches for the management of dyspnea in cancer patients will be examined based on randomized controlled studies. Roles and responsibilities of nurses in the management of dyspnea will be summarized in the last section.

Dyspnea is defined as a feeling of respiratory distress and difficult breathing; patients often describe this symptom as breathlessness, difficulty breathing, or shortness of breath.[1] Prevalence of dyspnea in cancer patients has been reported to be $21-90 \%$, depending on the stage of the cancer, and is reported more frequently in the last 6 weeks of life.[2,3] One of the most frightening and discomforting symptoms of cancer, dyspnea initially occurs in patients only during periods of activity. Inten- sity and frequency of dyspnea increase with progress of the disease, restricting patient's daily life functions and causing a decrease in quality of life (QOL). $[1,4,5]$

Although the causes of common symptoms such as pain, nausea, and vomiting in patients with cancer are known, underlying causes of dyspnea are not completely understood.[3] The pathophysiology of dyspnea is multifactorial. This complex pathophysiology can be conceptually summarized into 3 components: (a) increase in respiratory effort in order to overcome a mechanical impairment (e.g. obstructive or restrictive lung disease, pleural effusion); (b) increase in proportion of respiratory muscle required to maintain normal workload (e.g. neuromuscular weakness, cancer cachexia, etc.); and (c) increase in ventilatory requirements (hypoxemia, hypercapnia, metabolic acidosis, anemia, etc.).[3,6] Causes of dyspnea in cancer patients can be grouped as directly or indirectly associated with cancer, induced by cancer treatment, and 
not associated with cancer.[7] Causes that are directly associated with cancer include: coverage of pulmonary parenchyma by primary or metastatic cancer, lymphangitis carcinomatosis, airway obstruction created by tumors, pleural tumors, pleural effusion, pericardial effusion, abdominal ascites, hepatomegaly, phrenic nerve palsy, multiple tumor microemboli, pulmonary leukostasis, and superior vena cava syndrome. Indirect causes of dyspnea associated with cancer include cachexia, electrolyte imbalance, anemia, pneumonia, pulmonary aspiration, pulmonary embolism, and paraneoplastic syndrome. Cancer treatment-induced causes of dyspnea include pulmonary fibrosis associated with chemotherapy, cardiomyopathy associated with chemotherapy, radiation pneumonia, and problems associated with surgery.[3] Non-cancer causes of dyspnea include asthma, chronic obstructive pulmonary disease (COPD), congestive heart failure, pneumothorax, anxiety, obesity, neuromuscular disease, and pulmonary vascular disease.[2,3]

\section{Diagnosis of Dyspnea}

There is no reliable, objective measure of dyspnea; patient self-report is only reliable indicator of dyspnea in clinical practice. Respiratory rate, oxygen saturation, and arterial blood gas (ABG) determinations neither correlate with nor measure dyspnea. Patients may be hypoxemic, but not dyspneic, or dyspneic, but not hypoxemic.[3] In clinical research setting, dyspnea may be measured in a number of ways. Functional assessment tools such as shuttle walking test and reading aloud of numbers have been validated. When functional assessment is difficult, tools such as the Visual Analog Scale (VAS)[8] and Borg[9] scale can be used. If focus is more on the impact of dyspnea on patient QOL, then a multidimensional tool should be considered such as the Lung Cancer Symptom Scale (LCSS), the Dyspnea Exertion Scale (DES), or breathlessnessspecific questionnaires such as the Cancer Dyspnea Scale (CDS).[5]

Accurate assessment is very important for clinical management of dyspnea. Patient history and physical examination are essential components. Drugs used by patient, smoking status, profession/occupation, and radiotherapy or chemotherapy treatments offer important clues for assessing dyspnea. In addition, patient psychosocial and spiritual stress must be well understood because of cognitive and emotional factors that can affect dyspnea. Use of pulse oximetry, complete blood count (CBC), and chest radiography methods in the physical examination, are helpful to clearly understand underlying pathophysiology. Although use of advanced tests such as $A B G$ analysis, pulmonary function tests, computed tomography (CT), echocardiography and ventilation-perfusion scanning increases medical costs, they are reported to be effective.[5]

\section{Management of Dyspnea}

Use of a multidisciplinary team approach, application of combination of pharmacological and non-pharmacological treatments, and education of patients/ relatives are needed to manage dyspnea with advanced cancer. $[4,5,10]$

\section{Pharmacological Approaches}

\section{Opioids}

Opioids have been shown to be the most effective pharmacological agents for symptomatic control of dyspnea. Morphine, fentanyl, hydromorphone and oxycodone are some of the most common opioids used to manage dyspnea. The mechanism of how these medications affect dyspnea is the same as that of pain relief: they reduce ventilation, anxiety, and the central perception of dyspnea (and pain) by binding to opioid receptors. [10] Morphine is the most common opioid used to relieve dyspnea, and can be administered via oral, parenteral, and nebulized routes. There is no standardized dose, administration scheme or administration method of morphine.[10] Morphine is reported to be effective in management of dyspnea without significant reduction in patient respiratory rate, respiratory effort, and oxygen saturation value.[2] Although oral or intravenous route is currently the treatment of choice, many randomized, controlled studies have been performed to determine efficacy of nebulized morphine. $[5,10]$ These studies have found that there is no significant difference between nebulized morphine and nebulized placebo; therefore, it is not recommended as first line treatment at this time.

\section{Anticholinergics and Beta2-Agonists}

Respiratory resistance increases due to bronchospasm, airway obstruction, effusion, and accumulation of secretions. Inhaled beta2-agonists or anticholinergics have been reported to assist in treatment of lung cancer patients who experienced sudden bronchospasm. 
Bronchodilators can reduce breathing effort. Anticholinergics administrated orally, subcutaneously, transdermally or inhaled such as glycopyrrolate, atropine, scopolamine, and hyoscyamine, are recommended to reduce secretions.[10]

\section{Anxiolytics}

Like opioids, benzodiazepines have been used to help provide relief from dyspnea in cancer patients. $\mathrm{Al}$ though their effects do not directly change breathing, they are effective for anxiety, panic and fear symptoms often associated with dyspnea. There is a lack of evidence regarding the effect of benzodiazepines in the management of dyspnea; however, lorazepam, diazepam, and midazolam have been reported to be effective in reducing anxiety-induced dyspnea.[2,5] Benzodiazepines, which exhibit their effects on gamma-aminobutyric acid (GABA) receptors, can be used for sedative, hypnotic, muscle relaxant anxiolytic, and anticonvulsant purposes. When benzodiazepines are administered in normal dose ranges they do not affect respiratory rate, but high doses are reported to suppress ventilation rate slightly. Navigante and et al. (2006) performed a randomized, single-blind study using midazolam as an adjunct therapy to morphine. They found that the combination of this benzodiazepine with morphine had a positive effect on dyspnea with minimal effect on somnolence.[11] In contrast to these results, Simon and et al. (2010) stated that there is no evidence to support the efficacy of benzodiazepines in the palliation of dyspnea in their metaanalysis.[5,12] More randomized, controlled studies aimed at assessing the efficacy of benzodiazepines used in conjunction with other opioids need to be performed.

\section{Diuretics}

Furosemide is a loop diuretic agent used in treatment of heart failure, pulmonary edema, acid and edema. There has been recent literature on the efficacy of inhaled furosemide for treatment of dyspnea. Studies performed using inhaled furosemide have been small and uncontrolled, but results have been positive.[13,14] Wilcock et al. (2008) conducted a randomized controlled study and reported that nebulized furosemide had no impact on dyspnea with cancer patients and did not cause any side effects.[15] Despite participants stating that they felt relief from the dyspnea, there were no statistically significant results in another randomized controlled trial examining the effect of nebulized furosemide in the management of cancer-related dyspnea.[14] Larger, randomized and controlled studies need to be performed and their results analyzed before guidelines to use these medications in treatment of dyspnea can bee recommended.

\section{Non-Pharmacologic Approaches}

As physical, psychosocial, emotional, and functional factors influence the development of dyspnea, pharmacological approaches alone are not sufficient to manage it. Acupuncture, acupressure, neuromuscular electrical stimulation, external nasal dilator strips, pulmonary rehabilitation, regular exercise programs, use of supplemental oxygen and fan have been reported to manage dyspnea.[4,5,16-18] In addition, nurse counseling, effective respiratory-cough exercises, patient education programs, relaxation techniques, and coping strategies have been reported to make significant contributions to alleviating dyspnea. $[8,9]$

\section{Acupuncture and Acupressure}

Acupuncture is one of the oldest complementary treatments in the world and originated in China and other Asian countries. It has been argued that acupuncture stimulates sensory receptors in the body by increasing the level of analgesia or endorphins in the central nervous system (CNS) and provides sedation, supports the immune system, and maintains homeostasis.[19]

Acupuncture professionals argue that there are meridians, or energy paths, that link the different parts of the body and correspond to neurovascular connective tissue. According to a hypothesis, acupuncture stimulates the somatosensory system with the release of endogenous opioids. It is known that acupuncture causes a release of neurotransmitters, neuropeptides and other hormones, and produces a common effect on body functions leading to electrophysiological changes in different areas such as the autonomic nervous system (ANS), pituitary gland, hypothalamus and other parts of the brain.[20] Acupressure is another therapy technique that is similar to acupuncture; it is practiced by applying physical pressure with fingers, hand, palms, elbows, and knee to selected points on the body.[21] Considering the common effects, it is expected that acupuncture and acupressure would provide positive results for symptom management of cancer and cancer-related treatments.[20] 
It is reported that acupressure used for dyspnea significantly reduced dyspnea scores in patients with COPD. A randomized controlled study using patients with COPD compared sham acupressure and self-administered acupressure and reported that there was a $1 / 3$ reduction in VAS dyspnea scores of patients in the acupressure group and a $20 \%$ decrease in the placebo group (sham acupressure).[22] Another study conducted in patients with COPD suffering chronic dyspnea found that acupressure applied to the correct pressure points decreased dyspnea and anxiety, and also increased activity tolerance.[23]

In the literature, only 1 acupuncture study was conducted for dyspnea in cancer patients. Although this study had no control group, it supported the positive effects of acupuncture. In that study, 30 patients with cancer received a single session of acupuncture and mean VAS scores quickly decreased from 42 to 24 after 10 minutes of acupuncture intervention, and this effect continued for the duration of 6-hour follow-up. After the 6-hour follow-up, symptom scores returned to baseline values.[24]

\section{Pulmonary Rehabilitation and Exercise Programs}

Pulmonary symptoms including exercise intolerance, dyspnea, and fatigue are often encountered in patients with cancer. These symptoms originate from the nature of the disease itself or indirectly related causes, and lead to an increase in symptom burden in patients at later stages. Pulmonary rehabilitation is a multidisciplinary therapeutic approach that has beneficial effects on respiratory symptoms and combines patient education and psychosocial support. It has been reported that pulmonary rehabilitation improves exercise performance and physiological capacity in patients with lung cancer.[25,26]

Lack of muscle training in cancer patients with dyspnea results in the production of low rates lactic acid and exercise intolerance. Although exercise education is an important component of pulmonary rehabilitation, patients with dyspnea experience significant difficulties participating in such activities.[27] It has been stated that exercise programs used in advanced stages of COPD patients reduce difficulty of breathing and alleviate dyspnea. In addition, these exercise programs are thought to be effective for cancer patients with dyspnea. $[27,28]$
A pulmonary rehabilitation study conducted with lung cancer patients demostrated significant improvements in symptoms.[29] Morris et al. (2009) performed a study in patients with different types of cancer and reported that pulmonary rehabilitation training involving short aerobic exercises had been particularly effective for rehabilitation of pulmonary symptoms.[30] Wenzel's (2010) randomized controlled study comparing effects of exercise and routine activity stated that patients in the exercise group had lower emotional stress levels. That study also found that increased aerobic exercise was associated with reduced fatigue scores.[31]

\section{The Use of Neuromuscular Electrical Stimulus}

Neuromuscular electrical stimulus is an application that seeks to regain function of muscles by stimulating nerves in the muscles. Neuromuscular electrical impulses produce high-intensity muscle contractions by imitating low and high-intensity standard resistance exercise. These impulses encourage patients to continue treatment and progress by increasing muscle power with a passive process. Neuromuscular electrical stimulation studies have largely been conducted in patients with COPD.[32] Further studies in this area are needed to determine the effects on cancer patients with dyspnea.

\section{The Use of External Nasal Dilator Strips}

Adhesive bands containing a central elastic strip are often used to prevent snoring and to support breathing through the nose during exercise. For the first time, Neuenschwander et al. (2006) used external nasal dilator strips in patients with cancer with the goal of reducing dyspnea-induced cumulated effort of continuous breathing. Study results indicated that there was no serious side effect to use of nasal strips and most patients were willing to use these bands. In that study, the authors concluded that the bands would be effective in reducing fatigue in patients and would provide beneficial effects in the management of dyspnea.[17]

\section{Oxygen Therapy and the Use of Portable Fans}

Oxygen therapy is still one of the basic approaches used in the treatment of severe dyspnea and is often applied for long-term severe hypoxemia. Oxygen therapy is generally prescribed for patients who have lifespan of less than 3 months, and for severe hypoxemia in the terminal stage, although it restricts the level of activity. 
$[33,34]$ Room air and oxygen therapy were compared in a study conducted with 31 patients with lung cancer or other metastatic cancer, and stated that there was no significant difference between the groups in terms of dyspnea.[35]

In another randomized controlled study conducted with 12 patients with lung cancer and dyspnea, half of participants walked with oxygen-enriched air and the other half with medical air. Although the group receiving oxygen-enriched air needed less rest during the walk test, this difference was not statistically significant. Oxygen-enriched air caused a reduction in exercise-induced respiratory rate compared to the medi$\mathrm{cal}$ air, but there was no significant difference between groups in terms of oxygen saturation.[36]

Cold air applied directly to areas of the face such as cheeks, nasal mucosa, and pharynx, was seen to reduce dyspnea for the first time in the Schwartzste (1987) study. Although the effect of fan use in reducing dyspnea has not been explained clearly, it is accepted that cold receptors would change respiration rate by stimulating the trigeminal nerve and reducing sensation with sensory effect. Studies about the use of a fan for palliation of dyspnea encourage the evidence. There was a significant decrease in perception of dyspnea in a randomized controlled trial that examined the impact of a fan directly applied to the cheeks, conducted with 49 patients (15 participants diagnosed with cancer). [37] Current studies in the management of dyspnea show that traditional oxygen therapy is not superior to the use of a fan.[38,39]

\section{The Multidisciplinary Team Approach}

Despite recent advances in pharmacology, management of dyspnea in the last period of life is difficult. Progressive dyspnea indicates poor prognosis. It also creates need for sedation and hospitalization to provide symptom control in the last stage of life. The establishment of specialized palliative care can serve to train/ support health professionals as well as provide more comfort for patients at the end of life.[40] Specialized palliative care units serve to manage complex and uncontrolled symptoms, support patients, maintain patient QOL, provide means to discuss best options for patients and their relatives within the framework of end of life care, educate other clinics that treat terminal stage cancer patients, and to give a standard of care according to guidelines to other clinics.[41]

\section{The Roles and Responsibilities of Nurses}

Nurses are a key member of the palliative care team and have important roles and responsibilities in the care and treatment of cancer patients with dyspnea. Nurses can increase the level of independence in activities of daily living (ADL) and can provide important contributions to patient QOL through close monitoring, administering treatments, oxygen support, appropriate positioning techniques, pursed lip and diaphragmatic exercises, postural drainage principles, clearance of secretions, intermittent ventilation of patient rooms, using a fan, instruction in energy conservation techniques, encouragement of patients in daily exercises and applyingng adequate/balanced diet.[2,4,8]

Dyspnea may be frightening, not just for patients, but also for caregivers. As the disease progresses, the severity of dyspnea and increased stress levels in patients adversely affect the QOL of both patients and caregivers. Patient relatives or spouse experience serious anxiety and despair because of patient dyspnea suffering.[42] Caregivers often think that management of dyspnea is very challenging since they have no strategies for how to alleviate it.[43] When approaching dyspnea with a holistic perspective, the impact of dyspnea on the lives of caregivers should also be adressed. Nursing care plans should also focus on the anxiety level of patient relatives, as well as psychosocial stress of patients and caregivers.[41]

A randomized controlled study was conducted on 20 patients who had small cell and non-small cell lung cancer, had completed their chemotherapy and radiotherapy, and received 1-hour sessions from a nurse for 3-6 weeks that included breathing re-training; counseling; and relaxation, coping and adaptation strategies. The control group did not receive any intervention. Patients in the intervention group had significant improvement in dyspnea, dyspnea-induced stress level and functional capacity compared to the control group at 3 months. [29]

Consequently, nurses can provide significant contributions to management of dyspnea by eliminating the dyspnea-induced anxiety and fear that occur in patient and caregivers by applying behavioral and psychotherapeutic approaches, teaching effective-coping strategies, following patients at their home, making regular phone calls, giving advanced directives and supporting patients and their families in the critical decision-making process. [8] 
Conflict of interest: None declared.

\section{References}

1. Mercadante S, Casuccio A, Fulfaro F. The course of symptom frequency and intensity in advanced cancer patients followed at home. J Pain Symptom Manage 2000;20(2):104-12.

2. Akyolcu N. Kanserli hastalarda dispne ve hemşirelik bakımı. C.Ü. Hemşirelik Yüksekokulu Dergisi 2002;6(1):1-8.

3. Thomas JR, von Gunten CF. Clinical management of dyspneoa. Lancet Onco 2002;3(4):223-8.

4. Ben-Aharon I, Gafter-Gvili A, Paul M, Leibovici L, Stemmer SM. Interventions for alleviating cancerrelated dyspnea: a systematic review. J Clin Oncol 2008;26(14):2396-404.

5. Thomas S, Bausewein C, Higginson I, Booth S. Breathlessness in cancer patients - implications, management and challenges. Eur J Oncol Nurs 2011;15(5):459-69.

6. Paice AJ. Oncology nursing, assesment and clinical care: Symptom management. Miaskowski C, Buchsel P, editors. St. Louis: Mosby; 1999.

7. Reinke EF, Hoffman LA. Assesment and management of clinical problems: respiratory system. In: Lewis SM, Heitkemper MM, Dirksen S, editors. Medical surgical nursing. St. Louis: Mosby; 2000.

8. Bredin M, Corner J, Krishnasamy M, Plant H, Bailey C, A'Hern R. Multicentre randomised controlled trial of nursing intervention for breathlessness in patients with lung cancer. BMJ 1999;318(7188):901-4.

9. Moore S, Corner J, Haviland J, Wells M, Salmon E, Normand $\mathrm{C}$, et al. Nurse led follow up and conventional medical follow up in management of patients with lung cancer: randomised trial. BMJ 2002;325(7373):1145.

10. LeGrand SB. Dyspnea: the continuing challenge of palliative management. Curr Opin Oncol 2002;14(4):3948.

11. Navigante AH, Cerchietti LC, Castro MA, Lutteral MA, Cabalar ME. Midazolam as adjunct therapy to morphine in the alleviation of severe dyspnea perception in patients with advanced cancer. J Pain Symptom Manage 2006;31(1):38-47.

12. Simon ST, Higginson IJ, Booth S, Harding R, Bausewein C. Benzodiazepines for the relief of breathlessness in advanced malignant and non-malignant diseases in adults. Cochrane Database Syst Rev 2010;(1):CD007354.

13. Shimoyama N, Shimoyama M. Nebulized furosemide as a novel treatment for dyspnea in terminal cancer patients. J Pain Symptom Manage 2002;23(1):73-6.

14. Kallet RH. The role of inhaled opioids and furo- semide for the treatment of dyspnea. Respir Care 2007;52(7):900-10.

15. Wilcock A, Walton A, Manderson C, Feathers L, El Khoury B, Lewis M, et al. Randomised, placebo controlled trial of nebulised furosemide for breathlessness in patients with cancer. Thorax 2008;63(10):872-5.

16. Morris GS, Gallagher GH, Baxter MF, Brueilly KE, Scheetz JS, Ahmed MM, et al. Pulmonary rehabilitation improves functional status in oncology patients. Arch Phys Med Rehabil 2009;90(5):837-41.

17. Neuenschwander H, Molto A, Bianchi M. External nasal dilator strips (ENDS) may improve breathlessness in cancer patients. Support Care Cancer 2006;14(4):386-8.

18. Vickers AJ, Feinstein MB, Deng GE, Cassileth BR. Acupuncture for dyspnea in advanced cancer: a randomized, placebo-controlled pilot trial [ISRCTN89462491]. BMC Palliat Care 2005;4:5.

19. Rickards LD. Therapeutic needling in osteopathic practice: An evidence-informed perspect. International Journal of Osteopathic Medicine 2009;12(1):2-13.

20. Kasymjanova G, Grossman M, Tran T, Jagoe RT, Cohen V, Pepe $\mathrm{C}$, et al. The potential role for acupuncture in treating symptoms in patients with lung cancer: an observational longitudinal study. Curr Oncol 2013;20(3):152-7.

21. Hakverdioğlu G, Türk G. Akupressur. Hacettepe Üniversitesi Hemşirelik Yüksekokulu Dergisi 2006;43-7.

22. Maa SH, Gauthier D, Turner M. Acupressure as an adjunct to a pulmonary rehabilitation program. J Cardiopulm Rehabil 1997;17(4):268-76.

23. Wu HS, Wu SC, Lin JG, Lin LC. Effectiveness of acupressure in improving dyspnoea in chronic obstructive pulmonary disease. J Adv Nurs 2004;45(3):252-9.

24. Filshie J, Penn K, Ashley S, Davis CL. Acupuncture for the relief of cancer-related breathlessness. Palliat Med 1996;10(2):145-50.

25. Bobbio A, Chetta A, Ampollini L, Primomo GL, Internullo E, Carbognani P, et al. Preoperative pulmonary rehabilitation in patients undergoing lung resection for non-small cell lung cancer. Eur J Cardiothorac Surg 2008;33(1):95-8.

26. Spruit MA, Janssen PP, Willemsen SC, Hochstenbag MM, Wouters EF. Exercise capacity before and after an 8-week multidisciplinary inpatient rehabilitation program in lung cancer patients: a pilot study. Lung Cancer 2006;52(2):257-60.

27. Simon ST, Bausewein C. Management of refractory breathlessness in patients with advanced cancer. Wien Med Wochenschr 2009;159(23-24):591-8.

28. Bausewein C, Booth S, Gysels M, and Higginson I. Nonpharmacological interventions for breathlessness 
in advanced stages of malignant and non-malignant diseases. Cochrane Database of Systematic Reviews, 2008.

29. Corner J, Plant H, A'Hern R, Bailey C. Non-pharmacological intervention for breathlessness in lung cancer. Palliat Med 1996;10(4):299-305.

30. Morris GS, Gallagher GH, Baxter MF, Brueilly KE, Scheetz JS, Ahmed MM, et al. Pulmonary rehabilitation improves functional status in oncology patients. Arch Phys Med Rehabil 2009;90(5):837-41.

31. Wenzel J. Results of a home-based walking intervention for patients undergoing cancer treatment. 16th International Conference on Cancer Nursing. International Society of Nurses in Cancer Care (ISNCC), Georgia USA, 2010. Abstract available from. www. isncc.org/files/PDF/16th_ICCN_Abstract_Book.pdf (p. 24; 0-36).

32. Man WD, Kemp P, Moxham J, Polkey MI. Exercise and muscle dysfunction in COPD: implications for pulmonary rehabilitation. Clin Sci (Lond) 2009;117(8):28191.

33. Gallagher R, Roberts D. A systematic review of oxygen and airflow effect on relief of dyspnea at rest in patients with advanced disease of any cause. J Pain Palliat Care Pharmacother 2004;18(4):3-15.

34. McDonald CF, Crockett AJ, Young IH. Adult domiciliary oxygen therapy. Position statement of the Thoracic Society of Australia and New Zealand. Med J Aust 2005;182(12):621-6.

35. Bruera E, Sweeney C, Willey J, Palmer JL, Strasser F, Morice RC, et al. A randomized controlled trial of supplemental oxygen versus air in cancer patients with dyspnea. Palliat Med 2003;17(8):659-63.
36. Ahmedzai SH, Laude E, Robertson A, Troy G, Vora V. A double-blind, randomised, controlled Phase II trial of Heliox 28 gas mixture in lung cancer patients with dyspnoea on exertion. Br J Cancer 2004;90(2):366-71.

37. Abernethy AP, McDonald CF, Frith PA, Clark K, Herndon JE 2nd, Marcello J, et al. Effect of palliative oxygen versus room air in relief of breathlessness in patients with refractory dyspnoea: a double-blind, randomised controlled trial. Lancet 2010;376(9743):784-93.

38. Galbraith S, Fagan P, Perkins P, Lynch A, Booth S. Does the use of a handheld fan improve chronic dyspnea? A randomized, controlled, crossover trial. J Pain Symptom Manage 2010;39(5):831-8.

39. Cranston JM, Crockett A, Currow D. Oxygen therapy for dyspnoea in adults. Cochrane Database of Systematic Reviews, 2008.

40. Booth S. Improving the palliative care patients with intractable breathlessness. PhD Thesis, University of London, 2008.

41. Bausewein C, Booth S, Gysels M, Kühnbach R, Haberland B, Higginson IJ. Understanding breathlessness: cross-sectional comparison of symptom burden and palliative care needs in chronic obstructive pulmonary disease and cancer. J Palliat Med 2010;13(9):1109-18.

42. Booth S, Silvester S, Todd C. Breathlessness in cancer and chronic obstructive pulmonary disease: using a qualitative approach to describe the experience of patients and carers. Palliat Support Care 2003;1(4):33744.

43. Gysels MH, Higginson IJ. Caring for a person in advanced illness and suffering from breathlessness at home: threats and resources. Palliat Support Care 2009;7(2):153-62. 